J. Appl. Numer. Optim. 2 (2020), No. 1, pp. 1-2

Available online at http://jano.biemdas.com

https://doi.org/10.23952/jano.2.2020.1.01

\title{
EDITORIAL \\ A SPECIAL ISSUE FOCUSED ON SUPERIORIZATION VERSUS CONSTRAINED OPTIMIZATION: ANALYSIS AND APPLICATIONS
}

In many mathematical formulations of significant real-world technological or physical problems, the objective function is exogenous to the modeling process which defines the constraints. In such cases, the faith of the modeler in the usefulness of an objective function for the application at hand is limited and it is probably not worthwhile to invest a great effort in reaching an exact constrained minimum point. This is a major justification for using the superiorization method for practical applications.

The superiorization methodology can be thought of, in some cases, as lying between feasibilityseeking and constrained minimization. It is not quite trying to solve the full-fledged constrained minimization problem; rather, the task is to find a feasible point which is superior (with respect to a target function value) to one returned by a feasibility-seeking only algorithm.

This special issue brings together seven articles on recent advances in the superiorization methodology and related perturbation resilient algorithms. The results present new approaches to challenging mathematical problems and applications in various fields such as variational inequalities, fixed point theory, parameter estimation and learning and inverse problems. A summary of these articles in alphabetical order is presented next.

M. Abbasi and T. Nikazad, in the paper entitled Superiorization of accelerated cyclic subgradient methods, introduce a block version and a perturbed block version of the accelerated cyclic subgradient projections method with constraints and illustrate the performances of the methods in the field of computed tomography.

Y. Censor, S. Petra and C. Schnörr, in the article entitled Superiorization vs. accelerated convex optimization: the superiorized/regularized least-squares case, study and compare the superiorization and optimization approaches for the reconstruction problem of superiorized/regularized least-squares solutions of underdetermined linear equations with nonnegativity variable bounds.

(C)2020 Journal of Applied and Numerical Optimization 
A. Gibali and M. Haltmeier, in the contribution entitled Superiorization and regularization and of inverse problems, combine the superiorization methodology with iterative regularization methods and show that the superiorized version of the scheme yields again a regularization method, however accounting for different prior information.

G. Herman, in the contribution entitled Problem structures in the theory and practice of superiorization, identifies the mathematical essence of the superiorization methodology.

T. Humphries, M. Loreto, B. Halter, W. O'Keeffe, and L. Ramirez, in the paper entitled Comparison of regularized and superiorized methods for tomographic image reconstruction, compare two approaches to image reconstruction in computed tomography (CT) which incorporate penalty functions to improve image quality in the presence of noisy data.

The objective of the paper entitled Inexact orbits of set-valued nonexpansive mappings with summable errors by S. Reich and A. Zaslavski, is to study the convergence of inexact iterates of a set-valued nonexpansive mapping which acts on a metric space in the case where the errors are summable.

A. Zaslavski, in the paper entitled Three extensions of Butnariu-Reich-Zaslavski theorem for inexact infinite products of nonexpansive mappings, studies the asymptotic behavior of inexact infinite products of nonexpansive mappings which transform a nonempty closed subset of a complete metric space, under the presence of summable errors, and generalize the results known in the literature for nonexpansive self-mappings of a complete metric space.

We give our sincerest gratitude to all the authors who have contributed to this special issue and to all anonymous reviewers who thoroughly evaluated submitted manuscripts within a short period.

Aviv Gibali

ORT Braude College, Karmiel, Israel

E-mail address: avivg@braude.ac.il

Gabor T. Herman

City University of New York, New York, USA E-mail address: gabortherman@yahoo.com

Christoph Schnörr

Heidelberg University, Germany

E-mail address: schnoerr@math.uni-heidelberg.de 